\title{
A Mobile Based Integrated Outage Management System
}

\author{
Nampuraja Enose ${ }^{\mathrm{a}}$
}

\begin{abstract}
This paper proposes an integrated outage management system based on mobile technology for managing utility outages to deliver an agreed standard of service (sos). This outage management system is built on the needs of a utility on client-server architecture. The client application (preferably mobile based) allows users to $\log$ an outage complaint with details like location, time of detection, type of outage and extent of outage. The application has built-in menus to narrow down to the closest relevance and also permits inclusion of other details using sophisticated mobile applications. Each complaint is uniquely tagged and directed to the server. The server application is built on a layered architecture that can intelligently assign it to the respective group which has an identified owner, based on the details like type of utility, location, time etc. All the child complaints are identified with the parent complaint making it easier to handle. The intelligence and analytics layer identifies the parent-child relationship and other relationships where cross-sector relationships are involved. This intelligence therefore makes an integrated outage management system for all infrastructure services. This application can also be enhanced to monitor social media interactions to capture outage discussions. The mobile based application makes it easier for the customers to quickly log a complaint and track its status real-time. The other benefits of this system include: reduced outage durations, faster restoration based on location predictions, reduction in outage frequency, accurate and real-time outage and restoration information, extended asset and service life, reduction in customer complaints and improved customer satisfaction.
\end{abstract}

Key words: Public infrastructure; Integrated systems; Monitoring and control; Outage management; Mobile technology; Layered architecture

\section{Introduction}

The public infrastructure, the combination of fundamental systems, which is made up primarily of water utilities, sewer lines, electricity grids, telecommunications, transportation and housing, has a large impact on society. It is fundamental for economic prosperity and plays a vital role in determining quality of life. Therefore, one of the key responsibilities is to ensure a good public infrastructure and efficient infrastructure management that can boost productivity and growth that keeps the economy healthy. At the same time, it is no secret that most of this infrastructure was built in the 20th century and is aging and failing, and the utilities are struggling to efficiently manage them, especially their lack of reliability, safety and efficiency. While there has been huge investment to improve the physical infrastructure, comparatively less attention has been paid by the majority of the utilities in managing the systems and maintaining

\footnotetext{
${ }^{a}$ nampuraja_enose@infosys.com Centre of Innovation for Tomorrow's Enterprise (CITE), Infosys Labs, Infosys Limited, Bangalore, 560100. India.

http://dx.doi.org/10.14453/isngi2013.proc.64
} 
the services they promise to deliver. In fact, most of the utilities still manage and control the existing infrastructures with manual processes. On the other hand, the changing climatic conditions, rapidly increasing population, disruptive technology adoption, the quest for sustainability and critical need to improve living standards is turning the spotlight firmly on building advanced infrastructure as an essential component of a comprehensive economic strategy. This is building up massive networks of intelligent infrastructure systems, with information and sensing technologies with complex interactions and interdependencies between individual systems. The systems for transmitting and processing information have also expanded and complicated the infrastructure network, beginning with telephone lines and now encompassing all sorts of IT and telecommunications systems. Therefore, one of the biggest challenges of the 21 st century is to better manage these complex "system of systems" and leverage on their capabilities for improved efficiency and better utility, which is critical for driving and sustaining economic growth.

This paper therefore proposes an integrated outage management system based on mobile technology which leverages the intelligence in infrastructure systems to help utilities better manage outages and deliver on the agreed standard of service.

\section{Outage management system}

Outage in critical infrastructures and their associated systems and networks cause interruption of critical services to users. Quite often these infrastructure systems and networks are taken for granted, but a disruption of one of those systems can have dire consequences. In addition, these systems encounter high frequency outages caused by weather, component wear and myriad other reasons, and the need to reduce outages and restoration time has become critically important. However, most of the utility companies are still grappling with outdated paper systems and traditional tools that require a high-degree of human interpretation and manual processing. Therefore, utilities have failed in efficiently managing these systems and maintaining the services they promise to deliver. These concerns along with the constantly growing customer requirements on the quality of service have made outage management systems a critical tool for reducing the magnitude and/or duration of disruption in services.

\section{A. Integrated outage management system}

One the principal reasons that some of the issues have not been answered satisfactorily is the lack of harmonised measures in managing the infrastructure and services, reckoning the infrastructure industry as a whole. Infrastructure stakeholders have focused on sector specific solutions thereby significantly missing the impacts and benefits that can be identified by assessing the inter-dependencies between these sectors. An integrated outage management system is therefore aimed at leveraging this unique opportunity by enabling cross-sector interactions between the primary infrastructure sectors and their associated systems. This system is, therefore a first step towards building a unified system that functions collaboratively and synergistically in responding to outages. 


\section{B. The Architecture}

The outage management system is built on a multilayered architecture that uses different layers for performing the different functionalities of the system. The following layers explain the building blocks of the system in detail.

\section{i. User access layer (client application)}

User access layer is where the user can directly log an issue on disruption or outage of a service from a specific service provider - like power blackout, telephone service disruption, sewage overflow and pipeline leakage. This can be initiated using a simple mobile application, which will have a form to open a new ticket. To make it user friendly and to maintain a standard pattern of information detail, a scroll down option would be made available for the different data inputs in the form. The user can, accordingly, fill the form with details like type of service disruption, location details, time of detection, type of outage and extent of outage. The standardization of information increases system usability and field-service productivity. The mobile application has built-in menus to narrow down to the closest relevance and also permits the inclusion of more details using sophisticated mobile applications like image or video. The additional information will provide service engineers with more detail for quickly responding to outages.

The user friendly interface allows the user to quickly open tickets and submit details on the outage. Once the filled form is submitted by the user a complaint ticket with a unique id is generated. The unique ticket is then sent to the data base layer. Any update to the ticket will be made available real-time at the mobile application or updated to the user via his/her preferred mode of communication - SMS or email or both. This overcomes the critical disconnect of lack of real-time field information, with most of the OMS systems today. Some of these systems provide updates based on historical data leading to stale information, leaving a set of frustrated customers who repeatedly have to call back for updated information. Real-time update is, therefore, very critical for users because they not only want to be assured that their utility is aware of their outage, but they also know that someone is working on the problem. Since quite often these are critical service disrupting outages, they also expect real-time information regarding the repair progress, the estimated restoration time and they are quite interested on the cause for the outage.

The user-access layer can also be provisioned to receive public complaints over an SMS, from public bulletin boards or through posts from social networking websites, like Facebook and Twitter. In these cases, these alerts are received by the service desk that can manually open a ticket with the basic details available. Once the ticket is opened, any update on a ticket from the field will in turn be updated real-time back to the users via the respective channels - SMS, public bulletin boards or the respective social networking sites. The service desk can also connect legacy systems and traditional sets of tools like Interactive Voice Response (IVR) and High Volume Call Answering (HVCA) systems.

\section{ii. Data base layer}

Data base layer is the centralized database of the integrated outage management system. It is organized to handle large quantities of information by systematically accessing, storing, securing 
and managing it. The accuracy of the system completely depends on the integrity of the database layer. The central database performs the following functionalities:

- Logs the user complaint received from the user access layer to the centralized database for all type of services.

- Stores the complete details of customers - the customer database, their location details, the type of services provided, class of customers (if any) and the SLA for different services.

- Stores the details of field service engineers - list of field engineers, expertise level, maintenance schedule, leave calendar and location details.

- Stores the details of infrastructure - the asset database, the unique identifiers, the network mapping, aging of assets and the history of failures.

- Has details of all the services provided by the different infrastructure sectors, the interdependencies between these sectors, cross-sector interactions details, maps of service territory, GIS based routing and entire network map. The field service engineers would therefore have access to GIS based digital maps which are up-to-date and enabled with new routing functionality.

This therefore forms the central repository for the outage management system and is the source database for the analytics layer to build intelligent analytic applications using this information.

\section{iii. Intelligence \& Analytics layer}

The Intelligence and Analytics layer with strong analytical capabilities is the core of the integrated outage management system. It is built with analytical components which intelligently analyses the data retrieved from the underlying database layer and aids service providers and field engineers in quick decision making. The primary function of this layer is to take intelligent decisions by continuously interacting with the relevant data residing at the database layer. As field service functions become more complicated, service providers are today forced to implement realtime field service analytics to maximize workforce productivity, adhere to service-level agreements and achieve service excellence. It performs the following functionalities:

- Analyses the customer tickets to validate if multiple tickets are opened for the same issue/outage.

- Builds outage details as charts and trend lines to explain the dimensions of current outages, the related outages (in case of multiple outages for the same issue) and the cross-sector inter-dependencies between different infrastructure sectors.

- Relates the outages to the network map showing all paths impacted by the outage (calculating database). It shall also show all other outages that impact the path $\operatorname{limit}(\mathrm{s})$ for the duration of the outage.

- Analyses the network map and does equipment identification mapping to identify all the equipment IDs/Asset IDs impacted by the outage. Each of the identified equipment is shown on the outage summary display. The Service desk then uses this information to proactively inform the affected customers and details impact and restoration. 
- Analyses the outages and calculates the forecasts restoration time/MTTR based on the historical data, SLA's and dimensions of current outages. This enables intuitive reporting.

\section{iv. Administration layer}

Administration layer is the operational layer of the outage management system. It uses the insights provided by the intelligence and analytics layer and executes on them. At administration layer the following set of functionalities are performed:

- The administration layer has a ticket management console/dashboard which is available to the administrator and the field service engineers. Tickets are managed from this console. It also enables alert mechanisms based on the SLAs.

- If multiple tickets are opened for the same issue, a parent ticket is opened and all the related customer tickets are assigned as child tickets to this parent ticket. This could be a single service outage or multiple services affected for a single event, thereby building a cross-sector integrated management system and avoiding duplication of work. Quite often a single event, like an uprooted tree at a public place, could impact multiple services like electricity, telecommunication, transportation, water and sewage lines - which need a single parent ticket to be managed.

- A field service engineer will always work on a parent ticket (if available for an outage) and any real-time update that is done on the parent ticket is, at the same time, updating all the child tickets. This also helps in overcoming the issue of multiple service engineers assigned with different tickets concerning the same issue.

- Tickets are normally self-assigned by the field service engineers to themselves based on their availability and expertise. In case the tickets are in queue and are close to violating the SLA for ticket assignment, the administrator can manually assign the ticket to the most relevant service engineer.

- The admin layer connects with the service desk for real-time updates to the users for those tickets manually opened by them - via SMS, public bulletin boards or the respective social networking sites. The service desk can also connect legacy systems and traditional sets of tools like Interactive Voice Response (IVR) and High Volume Call Answering (HVCA) systems.

- The admin layer is responsible for automations (as applicable) using the insights from the intelligence and analytics layer, for superior application performance.

- The admin layer manages the closure of the tickets. Once the ticket is closed, the closure information along with closure comments is updated real-time in the tickets and therefore with the customer. It is also sends to the service desk to update the customer on the manual tickets opened by them.

\section{Conclusion}

This is definitely not a system that will eliminate outages. No systems can accomplish that vision. However, this system, when implemented rightly, can increase utilities' ability to identify outages, increase efficiency in prediction, reduce outage response time, forecast restoration times, communicate accurate information to customers and accelerate the pace of repairs. It also 
makes it a "one-stop" cross-sector solution to handle the outages of different infrastructure systems and their associated networks.

\section{References}

${ }^{1}$ Straw Proposal (Aug 5, 2013). Outage Management System Replacement, California ISO, Shaping a renewed future, August 2013. 